\title{
Electroencephalographic Correlates of Psychometry
}

\author{
John A. Marks and Johann Schutte \\ Department of Psychiatry, Gisborne Hospital, Gisborne 4010, Newzealand
}

\begin{abstract}
Berger's "window on the mind" [1] has, with modern computing power, become a possibility. A portable electroencephalograph was used to demonstrate a correlation of personality with brain waves. In principle, this could make it as useful a clinical tool for psychiatrists as the stethoscope is for physicians. Besides clinical use, the method could be a cheap, efficient way of investigating the effects of psychopharmaceutical drugs.
\end{abstract}

Key words: Electroencephalograph, psychometry, personality, clinical tool, psychopharmaceuticals.

\section{Introduction}

At the 2012 meeting of the RANZCP in Tasmania, Professor Mark Solms of Cape Town argued that Freud's theories of psychology are corroborated by modern studies in neurochemistry. In particular, the reticular formation ( $\mathrm{RF}$, formatio reticularis) of the brain stem is responsible, via three main neurochemical agents, viz. dopamine, noradrenalin and 5-hydroxy-tryptamine, for consciousness. $\mathrm{He}$ demonstrated this with videos of a small child cuddling her infant sibling, lying on a bean-bag. Clearly the child was conscious and happy. Then Professor Solms showed an X-ray of her skull showing she was anencephalic.

It seems clear, he concluded, that consciousness arises from activity in the RF. Subsequent learning, via the cerebral cortex, is unconscious and has to be imported into consciousness and either recorded in the hippocampus or remembered by gradual structural modification [2] of cortical connections. On this basis, Freud is right but what he called "the unconscious" is conscious and, indeed, following his talk Professor Solms held a workshop entitled "The Conscious Id" [3].

Conversely, it is cortical processes that are

Corresponding author: John A. Marks, M.B., Ch. B., research field: clinical psychiatry. E-mail: johnmarks9@hotmail.com. unconscious until they are brought into the "theatre" of the affect-laden, "conscious id". It is held that the varying activity of dopaminergic, noradrenergic and tryptaminergic neurons in the RF produce the affective quality of conscious experience. The dispositions of personality, the basic affective outlook, may thus be contingent upon the "play" of the relative strengths of dopaminergic, noradrenergic and tryptaminergic activity and that suitable functions of these activities may correlate with the psychometric dimensions of openness $(\mathrm{O})$, extraversion $(\mathrm{E})$ and neuroticism $(\mathrm{N})$ respectively.

Hypothesis: Personality is manifested by ongoing activity of the biogenic amines of the brainstem which, in turn, tonically influence the electrical activity of the brain.

It is the purpose of this paper to demonstrate an electroencephalographic correlation with these psychometric variables.

\section{Methods}

Eighty subjects had four minutes of their electroencephalograms recorded, two minutes with eyes open and two minutes with eyes closed, the order being randomized. A 5-channel portable, wireless electroencephalograph (measuring $2 \times 8 \times 12 \mathrm{~cm}$ ) was used, the "Optima-4" manufactured by Neurobit of Danzig, Poland. Recordings were made from the vertex 
$(\mathrm{Cz})$, left frontal pole (Fp1) and right frontal pole (Fp2), with reference to the left auricular lobe and earthed through the right auricular lobe. This is illustrated in the video link [4].

The eighty subjects comprised sixty psychology undergraduates and twenty psychology outpatients on no drugs. All subjects completed the OCEAN psychometry questionnaire [5]. Eight subjects were eventually not included either because they were found to be on prescribed drugs or had not completed the psychometry questionnaire.

At the three active electrodes, Fp1, Fp2 and $\mathrm{Cz}$, the voltages ( $V_{1}, V_{2}$ and $V_{3}$ respectively) were recorded by John Marks in real time and the power spectrum of each subject's Berger rhythm was similarly recorded as $\mathrm{P}_{1}, \mathrm{P}_{2}$ and $\mathrm{P}_{3}$. Electroencephalograms were digitally recorded at $1,000 \mathrm{~Hz}$, yielding $1.92 \times 10^{7}$ bits of information.

Subjects were controlled for handedness using a 12-item questionnaire [6] and each subject's completed, 240-item NEOAC-PI-R (1992) psychometric questionnaire (herein referred to as OCEAN psychometry) was scored by Johann Schutte.

\section{Analysis}

A huge range of variables was available for examination.

(1) To restrict the field, it was decided first to establish a proof of principle and correlations were sought between linear combinations of the power spectra and OCEAN scores using Pearson's correlation coefficient. Very many more investigations upon a MATLAB platform are available for future researchers. In particular, application of the Bonferroni correction for multiple comparisons could elicit "true" significances.

With the eyes closed sample $(n=72)$, the power spectra recorded at the three electrodes and the OCEAN scores were organized as arbitrary linear combinations: $a \mathrm{P}_{1}+b \mathrm{P}_{2}+c \mathrm{P}_{3}$ for the power spectra and $x \mathrm{~N}+y \mathrm{E}+z \mathrm{O}$ for the psychometries
The coefficients $a, b, c$ and $x, y, z$ were varied integrally between -5 and +5 , yielding $11^{6}(1,771,561)$ comparisons.

(2) Poincaré transforms were also plotted of $\mathrm{V}(t)$ against $\mathrm{V}(t+\theta)$ for each electrode of each patient. In particular, Poincaré transforms with $\theta=\pi / 4$ were plotted of the voltages $V_{1}, V_{2}$ and $V_{3}$.

Geometrical measurements were made of various parameters of the resulting attractor patterns and these were compared with the OCEAN psychometric scores.

Johann Schutte rated the plots as "tight", "clustered" or "dispersed" and, given one subject each that he knew clinically as indubitably $\mathrm{N}, \mathrm{E}$ or $\mathrm{O}$ respectively, was asked to classify blind another thirty of the Poincaré plots.

\section{Results}

(1) 119 significant correlations were found.

The following were significant correlations of the power spectra of the Berger rhythm with OCEAN (eyes closed) (Table 1).

(2) Of the Poincare plots, 11 out of $11(100 \%)$ were correctly predicted as N. 14 out of $16(87.5 \%)$ were correctly predicted as O but only 1 out of $3(33 \%)$ were correctly predicted as E. Both erroneous Os were Es but this rudimentary criterion clearly distinguished $\mathrm{N}$ from non-N.

Table 1 OCEAN vs. Berger power.

\begin{tabular}{llll}
\hline OCEAN & Electrode & Pearson & $p$ \\
\hline C4: achievement & Fp2 & 0.3595 & 0.0011 \\
striving & Fp2 & 0.3567 & 0.0012 \\
C2: order & $\mathrm{Cz}$ & 0.3424 & 0.0019 \\
O2: aesthetics & $\mathrm{Cz}$ & 0.3401 & 0.002 \\
O3: feelings & $\mathrm{Fp} 2$ & 0.3075 & 0.0055 \\
C5: self-discipline & $\mathrm{Fp} 2$ & 0.2865 & 0.01 \\
C: conscientiousness & $\mathrm{Fp} 2$ & 0.2789 & 0.0123 \\
O: openness & $\mathrm{Fp} 1$ & 0.2592 & 0.0202 \\
E1: warmth & $\mathrm{Fp} 2$ & 0.2537 & 0.0232 \\
N6: vulnerability & $\mathrm{Fp} 1$ & 0.2484 & 0.0263 \\
A3: altruism & $\mathrm{Fp} 2$ & 0.2426 & 0.0301 \\
E2: gregariousness & $\mathrm{Fp} 2$ & 0.2369 & 0.0344 \\
N3: depression & $\mathrm{Fp} 2$ & 0.2227 & 0.0471 \\
\hline
\end{tabular}




\section{Discussion}

The apparent correlation of the power spectra with $\mathrm{C}$ and $\mathrm{O}$ is notable: $\left(\mathrm{P}_{3}+3 \mathrm{P}_{2}\right)$ appears to predict $67 \%$ of $\mathrm{C} ;\left(\mathrm{P}_{3}+\mathrm{P}_{2}\right)$ predicts $33 \%$ of $\mathrm{O}$. There are also significant but decreasing correlations with $\mathrm{E}, \mathrm{N}$ and $\mathrm{A}$ as functions of $\mathrm{P}_{2},\left(\mathrm{P}_{1}+\mathrm{P}_{2}\right)$ and $\mathrm{P}_{2}$ respectively. Psychologically, $\mathrm{C}$ and $\mathrm{O}$ are reflections of awareness, $\mathrm{C}$ of internal awareness and $\mathrm{O}$ of external awareness and these are, therefore, encouraging indications of the predictive power of the electroencephalogram.

Amador et al. [7] argues convincingly that the electroencephalogram may be considered as the Fourier sum of the Berger rhythm ( $\alpha$-rhythm occipitally, $\tau$-rhythm temporally, $\mu$-rhythm over motor cortex, etc.) and all other activities which he calls the xi-process and which shows the $1 / f$ distribution of fractal noise. Furthermore, the Berger rhythm appears as self-consciousness develops. Its prominence in EEG studies has been well reviewed [8].

Buzsáki [9] notes that all other dominant rhythms in the electroencephalograms are related in frequency to that of the Berger rhythm by powers of $e$, i.e., they can never resonate. Transient phase-locking with the Berger rhythm may be the mechanism by which different sources of activity "enter" and "leave" consciousness, e.g., Ref. [10]. The duration of this transient phase-locking appears Gaussianly distributed about ninety milliseconds, the duration of Lehmann's "Zeitquantum" ("quantum of time" or "atom of thought") [11]. The sequence of zeitquanten gives rise to the subjective sense of "stream of consciousness" and is consistent with Gödel's argument that Newton's "universal flowing time" is subjective [12]. Thus, the conscious mental state and its behaviour over time, viz. the personality, may be consistently construed as the neurochemical manifestation of the electroencephalogram. Many studies suggest [13] disorder of this mechanism as the pathological basis of many psychiatric conditions.
Birnbaum [14] held that the protean presentations of psychopathology are influenced by what he called "pathoplastic factors" such as intelligence, injury and, above all, the premorbid personality. Psychiatric nosology is dogged by the difficulty of separating pathognomonic signs of a mental illness from the pathoplastic contributions due to premorbid personality. Meyer's "reaction types" [15] was an early attempt to accommodate this. Cloninger's [16] interpretation of OCEAN psychometry invites the hypothesis of the correlation of $\mathrm{O}, \mathrm{E}$ and $\mathrm{N}$ with the activity of the biogenic amines, dopamine, noradrenalin and 5-hydroxytryptamine respectively. These considerations extend to cultural influences upon the growing brain in childhood [17].

\section{Conclusion}

If demonstrated, it may be reasonably surmised that the electroencephalographic correlates of psychometry could be useful biomarkers of dopaminergic, noradrenergic and tryptaminergic activity for assessing psychopharmacological action.

The heuristic potential, economy and simplicity of this method make experiments to replicate or refute the hypothesis (that there exists a correlation between electroencephalographic parameters and OCEAN psychometry) well worthwhile.

This paper demonstrates the feasibility of this in principle.

As practical clinicians, we appeal to mathematically more sophisticated colleagues to bring this proposal to fruition.

\section{Acknowledgements}

We acknowledge the instruction, assistance and fruitful cooperation provided by Professors Ian Kirk and Waleed Abdulla of Auckland University and Dr. Vanessa Lim, EEG technician at the Department of Psychology.

This paper has also benefited from discussion with Professor G. Knyazev of Novosibirsk. 


\section{References}

[1] Berger, H. 1929. "On the Electrenkephalogram of Man." Archiv für Psychiatrie und Nervenkrankheiten 87: 527-70. (in German)

[2] Hebb, D. 1949. The Organization of Behaviour. New York: John Wiley \& Sons.

[3] Solms, M. 2013. "The Conscious Id." Neuropsychoanalysis 15: 5-19.

[4] Marks, J. 2012. "EEG Demonstration" YouTube. http://www.youtube.com/watch?v=IrYEUh8DGkw 2012.

[5] Costa, P., and Macrae, R. 1987. "Validation of the Five-factor Model of Personality across Instruments and Observers." Journal of Personality and Social Psychology 52: 527-70.

[6] Oldfield, R. C. 1971. "The Assessment and Analysis of Handedness: The Edinburgh Inventory" Neuropsychologia 9: 97-113.

[7] Amador, A. A., Pascual-Marqui, R. D., and Valdés-Sosa, P. A. 1990. "Spatiotemporal Properties of the $\alpha$ Rhythm." In Machinery of the Mind, edited by John, E. R., Harmony, T., Privhep, L. S., Valdés-Sosa, M., and Valdés-Sosa, P. A. Berlin: Birkhäuser, 59-90.

[8] Fingelkurts, A. Alexander, and Fingelkurts, A. Andrew 2004. "Book review of the Brain's Alpha Rhythms and the Mind." Clinical Neurophysiology 115: 1944-5.
[9] Buzsáki, G. 2006. Rhythms of the Brain. Oxford University Press, 113.

[10] Lehmann, D. 1990. "Brain Electric Microstates and Cognition: The Atoms of Thought." In Machinery of the Mind, edited by John, E. Berlin: Birkhäuser, 209-24.

[11] Palva, S., and Palva, J. M. 2007. "New Vistas for $\alpha$-frequency Band Oscillations." Trends in Neurosciences 30: 150-8.

[12] Yourgrau, P. 1999. Gödel Meets Einstein. Chicago: Open Court.

[13] Uhlhaas, P. J., and Singer, W. 2006. "Neural Synchrony in Brain Disorders: Relevance for Cognitive Dysfunctions and Pathophysiology." Neuron 52: 155-68.

[14] Birnbaum, K. 1923. The Building of Psychosis. Berlin: Julius Springer. (in German)

[15] Meyer, A., Winters, E. E., and Bowers, A. M. 1957. Psychobiology: A Science of Man. Springfield: Charles C Thomas.

[16] Cloninger, R. 1987. "A Systematic Method for Clinical Description and Classification of Personality Variants." Arch Gen Psychiatry 44: 573-88.

[17] Jilek, W. G. 2000. "Culturally Related Syndromes." In New Oxford Textbook of Psychiatry, edited by Gelder, M. G., Lopez-lbor, J. J., and Andreasen, N. Oxford: OUP, 1061-6. 